\title{
Lessons Learned from a Healthcare Associated Infection Tabletop Exercise, June 2017
}

\author{
Dana Elhassani and Rachel J. Ilic* \\ Epidemiology, Florida Department of Health in Pinellas County, Saint Petersburg, FL, USA
}

\section{Objective}

To assess healthcare facilities' level of readiness to respond to an HAI outbreak, the Florida Department of Health in Pinellas County (DOH-Pinellas) conducted an HAI outbreak tabletop exercise (TTX) on June 6, 2017. Other benefits of this TTX were to provide opportunities for collaborative learning, building community partnerships and evaluation of HAI preparedness activities in Pinellas County healthcare facilities.

\section{Introduction}

One in twenty-five patients in acute care hospitals develop at least one health care associated infection (HAI); this resulted in approximately 75,000 preventable deaths in 2011. Risk factors associated with developing HAIs include older patients, serviced at a large hospital, central catheter placement, receiving medical ventilation, and placement in a critical care unit. In Pinellas County, individuals 65 years of age and older comprise approximately $24 \%$ of the total population.

\section{Methods}

A line list of contact information for all long-term care facilities in Pinellas County was obtained from the DOH-Pinellas Environmental Health Program. An invitation to the TTX was sent to 234 health care and assisted living facilities. Of those invited, 35 individuals attended the TTX on June 6, 2017. The methods used to facilitate discussion included the four scenarios that addressed different stages of an outbreak investigation and a "force decision-making" framework. Following the course, a twelve-question evaluation was distributed. The first seven questions were based on a five-point Likert scale assessing the course's impact on knowledge and tabletop learning environment. The other five were open-ended and asked participants to elaborate on what they learned and provide feedback regarding the strengths and areas for improvement of the TTX.

\section{Results}

Exercise participants included infection control practitioners, safety officers, nursing supervisors, facility managers and epidemiologists from hospitals, assisted living and skilled nursing facilities, hospice, rehabilitation centers and health departments. Of the 35 participants, 30 completed the course evaluation for a completion rate of $86 \%$. For questions addressing HAI knowledge, participants strongly agreed that the tabletop exercise enhanced their understanding of infection control guidelines for HAIs. The question that received the lowest score of 4.3 was the perception that "I was able to develop tools for my agency's infection control guidelines." In the open-ended questions, themes regarding HAI knowledge, resources, response and policies were frequently mentioned, in addition to confusion over the health department's role and available resources during an HAI outbreak.

\section{Conclusions}

Participation and feedback during the TTX substantiated the importance of increased collaboration across organizations and opportunities for training on HAI outbreak response. Participants identified a need for an open forum to discuss best practices for HAI control and surveillance methods to help guide preparedness and response efforts. To address this need, DOH-Pinellas will create a HAI coalition which would aim to improve understanding of each facility's role in responding to an HAI outbreak.

\section{Keywords}

Healthcare Associated Infection; Coalition; Pinellas County

${ }^{\star}$ Rachel J. Ilic

E-mail: rachel.janssenilic@flhealth.gov 